\title{
Is Thermal Responsiveness Affected by Maternal Estrogens in Species with Temperature- Dependent Sex Determination?
}

\author{
Rachel M. Bowden Ryan T. Paitz \\ School of Biological Sciences, Illinois State University, Normal, IL, USA
}

\section{Keywords}

Fluctuating temperatures · Incubation · Sex determination/ differentiation $\cdot$ Turtle $\cdot$ Yolk estrogens

\begin{abstract}
In species with temperature-dependent sex determination (TSD), incubation temperatures regulate the expression of genes involved in gonadal differentiation and determine whether the gonads develop into ovaries or testes. For most species, natural incubation conditions result in transient exposure to thermal cues for both ovarian and testis development, but how individuals respond to this transient exposure varies and can drive variation in the resulting sex ratios. Here, we argue that variation in the timing to respond to temperature cues, or thermal responsiveness, is a trait needing further study. Recent work in the red-eared slider turtle (Trachemys scripta) has found that when embryos experience transient exposure to warm conditions (i.e., heatwaves), some embryos show high responsiveness, requiring only short exposures to commit to ovarian development, while others show low responsiveness, developing testes even after more extended exposures to warm conditions. We discuss how maternal estrogens might influence thermal responsiveness for organisms that develop under thermal fluc-

tuations. Examining the interplay of molecular responses to
\end{abstract}

karger@karger.com

(c) 2021 S. Karger AG, Basel

www.karger.com/sxd

Karger' more subtle thermal and endocrine environments may reveal significant insights into the process of sex determination in species with TSD.

(c) 2021 S. Karger AG, Basel

\section{Introduction}

All organisms are affected by environmental temperatures, and the effects of temperature can range from immediate alterations of biochemical processes to serving as a cue for complex biological processes. For the more complex processes (e.g., reproduction, emergence, migration), the effect of environmental temperatures on the underlying molecular processes must often be integrated over an extended period of time (i.e., days to months) before a response occurs. The timing of biological events in response to environmental temperatures is well studied as it relates to phenology, but the focus of most of these studies is at the population level and how climate change may influence these processes [Visser and Both, 2005; Amano et al., 2010; Shipley et al., 2020]. Much less work has been done to investigate the mechanisms underlying individual variation in the time it takes to respond to an environmental cue. 


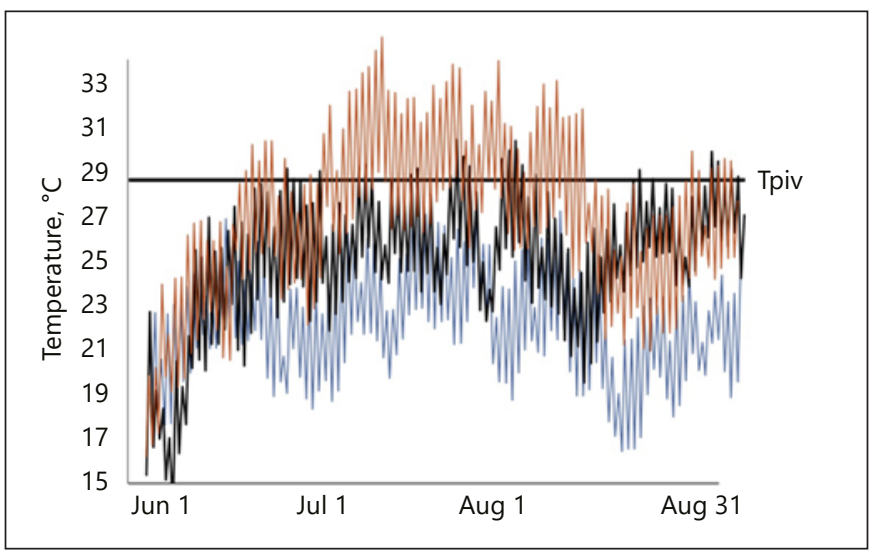

Fig. 1. Soil thermal traces from a cool (blue), average (black), and warm (red) year soil in central Illinois. The $\mathrm{T}_{\text {piv }}$ represents the temperature above which ovarian differentiation occurs in T. scripta. Adapted from Carter et al. [2018].

A wide variety of molecular mechanisms can influence how an organism responds to temperature. For example, protein phosphorylation [Abe et al., 2018], histone methylation/demethylation [Ge et al., 2018], and RNA splicing [Preußner et al., 2017] can all respond to temperature and subsequently alter gene expression programs that mediate the physiological response to temperature. At a mechanistic level, the integration of environmental temperatures often occurs through the accumulation of a biochemical signal that eventually reaches a threshold, initiating the biological process [Capovilla et al., 2017; Kinmonth-Schultz et al., 2019]. Because there are numerous components that underlie an organism's response to temperature, variation in any one component could hypothetically alter the amount of time it takes to respond to temperature. Variation in the time it takes an individual to respond to a temperature cue could have important ramifications on how, or even if, a physiological response occurs. Traits related to the timing of the response are understudied and yet may be critical for understanding why some individuals require only modest exposure to a temperature cue to initiate a response, while others need more extensive exposure to respond.

In nature, organisms receive mixed thermal signals as a result of transient exposure to thermal cues (Fig. 1) [Bock et al., 2020a; Fleming et al., 2020], and this may dictate whether or not they respond. In most environments, organisms experience both daily and seasonal variation in temperatures, but the majority of the work on how temperature affects molecular processes has been done under conditions with no or minimal thermal vari- ability [Matsumoto et al., 2013; Deveson et al., 2017; Politis et al., 2017; Ge et al., 2018]. One consequence of this experimental approach is that variation in the time it takes to respond to a thermal cue is masked. Given our rapidly advancing understanding of how temperature affects molecular processes, investigating how these processes are integrated under ecologically relevant conditions to coordinate complex biological processes is needed. Specifically, we need to understand if the time required to respond to an environmental cue, such as temperature, is a trait capable of responding to environmental variation. Temperature-dependent sex determination (TSD) is an ideal candidate system for examining time-dependent variability in responsiveness to thermal cues, or "thermal responsiveness", which for the purpose of this paper we define as the time it takes to commit gonadal fate in response to temperature cues. Incubation temperatures experienced during development drive the formation of an ovary or a testis in species with TSD, and as such, the outcome of individual responsiveness is effectively binomial. Nest temperature traces from a variety of species with TSD indicate that nests can experience thermal conditions that would promote either ovary or testis formation within the span of a single day [Carter et al., 2018; Valenzuela et al., 2019; Bock et al., 2020a]. Our recent work on the red-eared slider turtle (Trachemys scripta) has demonstrated that short exposures to warm temperatures can trigger ovarian development in some embryos [Carter et al., 2018], but this effect can be greatly reduced if the exposure to warm temperatures is not continuous [Breitenbach et al., 2020]. These results highlight the importance of understanding how some embryos respond to brief temperature exposures and help define thermal responsiveness as a trait that may underlie the effect of incubation temperatures on sex ratios in species with TSD. More specifically, we know that some individuals are highly responsive to short exposures to warm temperatures, while others require longer exposures to respond (Fig. 2), but we do not know the underlying drivers of this variation yet. A recent study found that, under thermal fluctuations, some genes associated with sex determination are capable of rapid responses to changing thermal cues [Bock et al., 2020b], which may provide a mechanism for variation in responsiveness. Since many of the genes involved in sex determination are regulated by temperature and estrogens, it is possible that factors such as maternal steroids might affect thermal responsiveness and that there may be underlying genetic variation in how different genes respond to both temperature and estrogens. Ultimately, variation in thermal respon- 
Fig. 2. Representative thermal conditions used to characterize variation in thermal responsiveness of T. scripta embryos. Embryos classified as having high responsiveness required only 5 days of exposure to warm temperatures, while those with low responsiveness required $11+$ days of exposure to trigger ovarian development. Based on Carter et al. [2018].

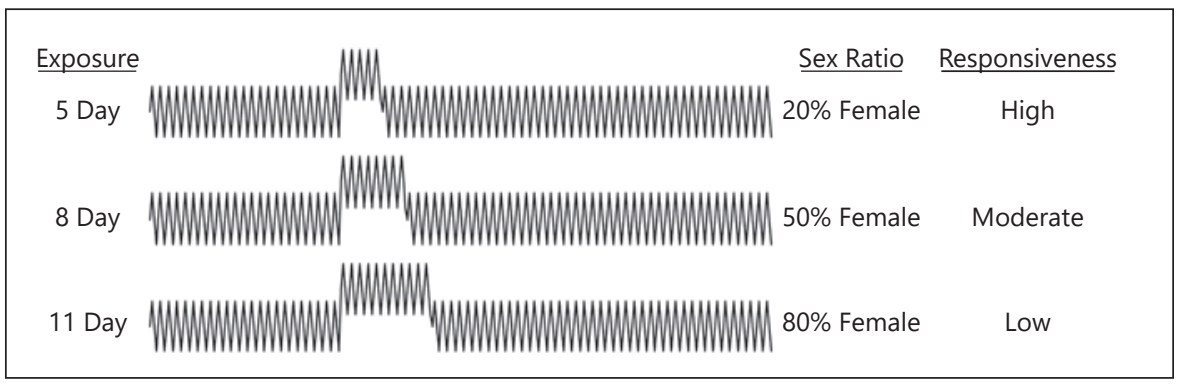

siveness may provide a means by which organisms can adjust to different climates through either genetic or nongenetic means.

\section{Constant Incubation Temperatures and Sex Ratios}

Reptiles with TSD face an uncertain future as climate patterns change, and at present, researchers are poorly equipped to predict how changes in environmental temperatures will affect sex determination in these thermally sensitive species. Although TSD is not a common mode of sex determination, it is known to occur in some fishes and is widespread in reptiles including some lizards, most turtles, and all crocodilians [reviewed in Bachtrog et al., 2014]. Depending upon the species in question, constant incubation at warmer temperatures may promote ovary development, while cooler temperatures promote testis development, or vice versa, and the transition between temperatures that promote one or the other is narrow [Bull, 1980]. The temperature that, under constant incubation conditions, produces a population-wide 50:50 sex ratio is known as the pivotal temperature $\left(\mathrm{T}_{\text {piv }}\right)$, and across reptiles with TSD, there is relatively little variation in $\mathrm{T}_{\text {piv }}$ at the population level [Bull et al., 1982; Carter et al., 2018; Carter AL et al., 2019]. Many hypotheses regarding the mechanisms underlying TSD are based on the concept that when incubation temperatures are either above or below $\mathrm{T}_{\text {piv }}$, the bipotential gonad accumulates a biochemical signal that must reach a threshold before gonadal differentiation is triggered [Deeming and Ferguson, 1989; Carter et al., 2018]. In T. scripta, ovarian differentiation is triggered at temperatures above $\mathrm{T}_{\text {piv }}$ and testis differentiation below $\mathrm{T}_{\text {piv }}$ (Fig. 1), but the accumulating biochemical signal is unknown. We propose that variation in the threshold for the biochemical signal and/or the rate of signal accumulation and loss would manifest itself as variation when sex becomes committed during development, with more thermally responsive individuals committing to gonadal fate earlier in development. Unfortunately, when incubation temperatures are constant for the entirety of development, timing-related traits are often masked because all embryos eventually reach the threshold for committing to a gonadal fate.

In species with TSD, the bipotential gonad is most sensitive to temperature during the middle third of development, the temperature sensitive period (TSP) [Mrosovsky and Pieau, 1991; Wibbels et al., 1991a]. In general, the TSP is described as the middle third of development because temperature manipulations earlier or later in development do not typically affect sex ratios [Valenzuela, 2001; Mork et al., 2014; Gómez-Saldarriaga et al., 2016]. It is hypothesized that early in development, the embryo is not yet competent to respond to temperature (i.e., the bipotential gonad is not yet present), while later in development gonadal fate has already been "determined" (i.e., gonadal differentiation has been initiated). Ultimately, the TSP represents the stages of development when sex can be determined, but it does not define precisely when sex is determined. While studies using constant incubation temperatures were extremely valuable for defining the range of temperatures that induce gonadal differentiation and providing an initial characterization of the stages of development for when these effects take place, a consequence of this heavy reliance on constant incubation conditions is that the results may have limited to no biological relevancy for most species that experience periods above and below $\mathrm{T}_{\text {piv }}$ on a regular basis during the TSP [Bowden et al., 2014]. To help redress this issue, many studies now use naturalistic thermal fluctuations to better approximate conditions organisms would experience in the field [Les et al., 2009; Carter AW et al., 2019; Valenzuela et al., 2019; Breitenbach et al., 2020; Hall and Warner, 2020]. 


\section{Fluctuating Incubation Temperatures and Sex Ratios}

We know that, in nature, nest temperatures fluctuate daily and that the frequency and duration of exposure to any particular thermal condition can be considered patchy (e.g., weather events that alter local thermal conditions). Studies using continuous fluctuating incubation temperatures (e.g., $28.5 \pm 3^{\circ} \mathrm{C}$ for the entirety of incubation) in the laboratory have demonstrated that for species where females are produced at warm incubation temperatures, temperature fluctuations lead to an increased production of female hatchlings [Georges et al., 1994; Les et al., 2007; Paitz et al., 2010; Valenzuela et al., 2019]. It is thought that this occurs because under fluctuating conditions, more development takes place during the time at female promoting temperatures. For example, if an embryo spends $12 \mathrm{~h}$ above $\mathrm{T}_{\text {piv }}$, it will experience more growth at these warmer, female producing temperatures than it will during the $12 \mathrm{~h}$ at cooler, male producing temperatures.

There is an increasing recognition that the sex determination response under natural incubation conditions is the result of a dynamic interplay between exposure to thermal conditions that, depending upon the species and pattern of TSD, would promote the development of testes and those that would promote the development of ovaries during the course of development [Carter et al., 2018; Valenzuela et al., 2019]. In particular, transient exposure to high temperatures such as those experienced during heatwaves or abnormally hot weather lasting at least 2 days have been critical for allowing us to quantify the time it takes the sex determination cascade to respond to temperature [Carter AW et al., 2018, 2019]. Using fluctuating temperatures in the laboratory and simulated heatwaves, we have demonstrated that embryos require a relatively short exposure to heat to trigger ovarian development. A heatwave of just 8 days produces a 50:50 sex ratio, and a heatwave of 15 days produces $95 \%$ females [Carter et al., 2018]. Exposing developing T. scripta eggs to 14-day heatwaves that fell mostly or completely within the TSP, we found that heatwaves starting between days 17-31 of development resulted in sex ratios of $>70 \%$ females, while heatwaves initiated on day 10 or 38 of development produced $\leq 12 \%$ females [Breitenbach et al., 2020]. Despite a strong effect on sex ratios, there were minimal effects of heatwave timing on average incubation duration (day 10, 72.4 days; day $17,72.6$ days; day $24,73.3$ days; day $31,74.1$ days; day 38, 75.4 days). These results highlight how the timing of the thermal cue can affect sex determination, while other traits are not affected.
In an effort to better understand if the molecular signals initiated by temperature are only accumulated with exposure to warm temperatures (i.e., ratchet) or if they can be gained and lost with exposure to variable temperatures, we coupled discontinuous exposure to a heatwave with cooler fluctuating temperatures. We found that the signal can be both gained and lost as thermal conditions change as evidenced by changes in the resulting sex ratios; a 1-day break in a heatwave was not sufficient to disrupt female development (50\% females), but a 3-day break resulted in only $12 \%$ females [Breitenbach et al., 2020]. It is worth noting that we only studied breaks starting on incubation day 24 , so whether breaks occurring earlier or later would elicit different effects is unknown. One important consequence for embryos experiencing thermal variability during development is that it appears to delay when sex is determined, likely because the bipotential gonad experiences less consistent thermal cues [Breitenbach et al., 2020]. By comparison, constant incubation temperatures likely initiate sex determination at the earliest stages possible because there are no conflicting thermal cues. The result is that experiments using constant incubation temperatures are studying sex determination at developmental stages that are very unlikely to be the same as when sex is determined under natural incubation temperatures (Fig. 3). Knowing when sex is determined in the field can facilitate the use of nest temperatures as a non-invasive metric for predicting sex ratios, which could be of great use in threatened or endangered species.

Early efforts to incorporate variable thermal conditions into a predictive metric resulted in the constant temperature equivalent (CTE) model, which provided an estimate of the temperature at which half of development occurs above and half of development occurs below [Georges, 1989]. Embryos that experience most of their growth above $\mathrm{T}_{\text {piv }}$ are predicted to develop as females, whereas those experiencing most growth below $\mathrm{T}_{\text {piv }}$ are predicted to develop as males in species where warmer conditions result in the formation of ovaries and cooler conditions result in the formation of testes. More recently, models incorporating the CTE have strengthened our ability to predict sex ratios. The weighted Cumulative Temperature Units (wCTU) model relies on the time (in hours) that embryos spend at temperatures above and below $\mathrm{T}_{\text {piv }}$, which are then weighted by the temperature-specific developmental rate [Valenzuela et al., 2019]. The daily duration with the CTE (DDC) model calculates whether daily temperatures reside above or below $\mathrm{T}_{\text {piv }}$, resulting in a day 


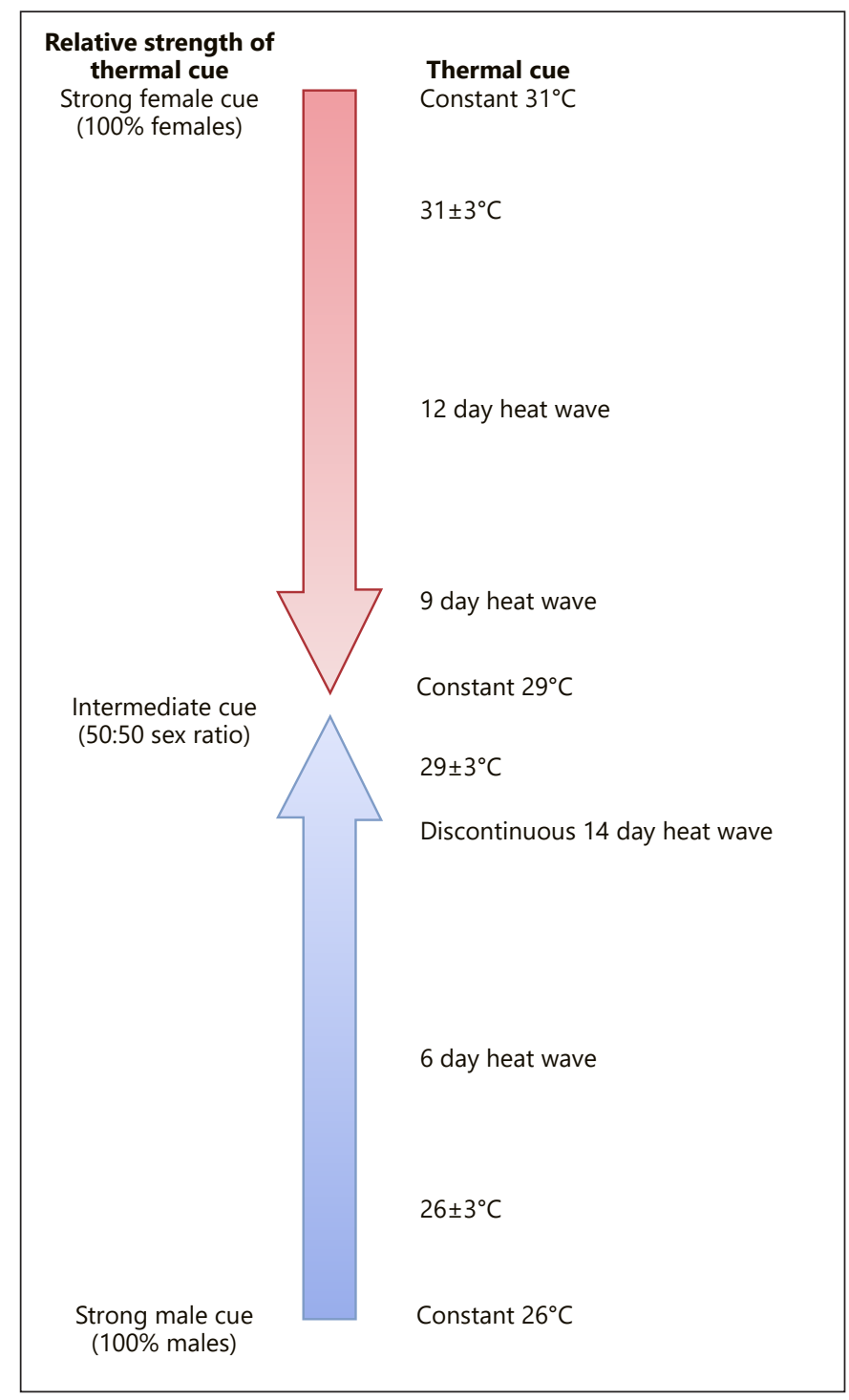

Fig. 3. Schematic representation of the effects of the relative strengths of thermal cues on sex ratios.

that is female or male producing, respectively [Carter et al., 2018]. This DDC model essentially counts the number of days at female-producing temperatures during the TSP and compares those days to the reaction norm to predict sex [Carter AW et al., 2019].

\section{Maternal Estrogens and Sex Ratios}

Temperature, however, is not the only factor known to affect sex determination in species with TSD. There is a lot of literature detailing how both exogenous and endogenous estrogens can promote the development of ovaries in species with TSD [Wibbels et al., 1991b; Paitz and Bowden, 2011; Carter et al., 2018], and we know that estrogen levels are not static across the season [Bowden et al., 2000, 2001; Paitz and Bowden, 2009, 2013; Paitz et al., 2012]. Most of the work on the effect of estrogens has used 17ß-estradiol, applied topically or injected at the start of the TSP [Wibbels et al., 1991b; Ge et al., 2018]. More recently, we have investigated the effects of major metabolites of $17 ß$-estradiol and found that they are also able to affect sex determination [Paitz and Bowden, 2011, 2013]. We detected that topically treating eggs with either estradiol sulfate or estrone sulfate at oviposition produced female-biased sex ratios. Further, in unmanipulated eggs, estrone sulfate is far more abundant than is 17ß-estradiol [Paitz and Bowden, 2013]. Because the evolution of TSD has taken place under conditions of thermal and endocrine variability, it stands to reason that to truly understand the complex, and likely subtle, effects of temperature and steroids on this process, we must take a new approach that integrates biologically meaningful thermal and endocrine conditions.

Many reptiles with TSD, including T. scripta, produce multiple clutches across the nesting season, and endogenous steroid concentrations in eggs vary seasonally, with estrogens, including estradiol sulfate, being significantly lower in early season clutches than in later clutches [Bowden et al., 2000; Paitz and Bowden, 2009, 2013]. This means that earlier clutches of T. scripta generally experience both cooler thermal conditions and lower estrogens during development, factors that should promote the production of males, while later clutches generally experience warmer thermal conditions and higher estrogens, promoting the production of females. In a recent study, we examined the interplay between temperature fluctuations and endogenous estradiol and found that, when exposed to the same thermal conditions, late season eggs produced up to $49 \%$ more female hatchlings than early season eggs when incubated under identical temperatures [Carter et al., 2017]. We have also examined the effect of exposing eggs to heatwaves of increasing durations during the TSP by incubating eggs at a baseline temperature of $27 \pm 3^{\circ} \mathrm{C}$ and then to a simulated heatwave of $29.5 \pm$ $3^{\circ} \mathrm{C}$ for 5-30 days before returning them to baseline. As expected, eggs that experienced longer exposure to a heatwave were more likely to become female, but surprisingly, we achieved a 50:50 sex ratio with just an 8-day heatwave, and late season eggs required significantly fewer heatwave days to become female [Carter et al., 2018]. These data further support the idea that there is an interplay between temperature and steroids in sex determination 
for species with TSD. We expect that the effects of estrogens would be most pronounced at intermediate thermal conditions.

\section{Cascade of Molecular Events in TSD}

"The business of sex determination is to activate 1 of the 2 developmental pathways and shut down the other" [Capel, 2017]. For vertebrates, gonadal differentiation involves activating a network of relatively conserved genes necessary to construct an ovary or testis, respectively [Capel, 2017], but there is a remarkable amount of variation in the factors that can activate these networks [Bachtrog et al., 2014]. Decades of work investigating how temperature and estrogens determine sex has provided a detailed characterization of the effects on gene expression patterns in developing gonads [Ramsey and Crews, 2009]. The most common approach has been to incubate eggs at $26^{\circ} \mathrm{C}$ to induce testis development or at $31^{\circ} \mathrm{C}$ to induce ovary development, sample embryos at equivalent developmental stages, and compare expression patterns [Matsumoto et al., 2013; Deveson et al., 2017; Ge et al., 2018]. Applying estradiol to eggs incubated at $26^{\circ} \mathrm{C}$ when they reach stage 15 of development (i.e., the start of the TSP in T. scripta) consistently results in the production of $100 \%$ female hatchlings, and this has been used to examine how estrogens influence gene expression [Ramsey and Crews, 2009; Matsumoto et al., 2013]. Many studies use qPCR to quantify expression, but several recent studies have also employed a transcriptomic approach to identify novel temperature-responsive genes that may be involved in the sex determining cascade [Yatsu et al., 2016; Deveson et al., 2017; Radhakrishnan et al., 2017]. In T. scripta, transcriptomic analysis has revealed that thousands of genes (i.e., over 4,000 sexually dimorphic genes at stage 15) differ when comparing gonads from embryos incubated at $26^{\circ} \mathrm{C}$ versus $31^{\circ} \mathrm{C}$ [Czerwinski et al., 2016]. These studies have helped to identify genes that are differentially expressed, as well as the relative timing of when those genes are expressed in a dimorphic manner, but only recently have we begun to understand how temperature actually activates these developmental pathways.

\section{Male Pathway}

A number of temperature sensitive processes, including calcium influx [Weber et al., 2020], protein phosphorylation [Weber et al., 2020], histone demethylation [Ge et al., 2018], and alternative splicing [Anand et al., 2008; Deveson et al., 2017], have all been implicated in transducing temperature cues into altered gene expression. In particular, this work has focused on how these processes regulate the expression of Doublesex mab3-related transcription factor 1 (Dmrt1), a critical regulator of testis development in mammals [Zhao et al., 2015], birds [Lambeth et al., 2014], and reptiles [Ge et al., 2017]. A series of constant temperature studies in T. scripta have demonstrated that temperature regulates the expression of Dmrt 1 through a series of biochemical and epigenetic processes. Under cool incubation temperatures $\left(26^{\circ} \mathrm{C}\right)$, expression of the histone demethylase $(K d m 6 b)$ begins to rise at the beginning of the TSP [Ge et al., 2018]. Knocking down Kdm6b expression results in ovarian development [Ge et al., 2018 ], suggesting that $K d m 6 b$ is necessary for testis development. To complicate matters further, male producing temperatures induce the retention of an intron in the $K d$ $m 6 b$ transcript [Deveson et al., 2017]. When expressed, $K d m 6 b$ induces Dmrt1 expression by demethylating histones at its promoter [Ge et al., 2018], and Dmrt1 induces the expression of sex-determining region on Y chromosome-box 9 (Sox9), another highly conserved transcription factor involved in testis development of vertebrates [Matsumoto and Crews, 2012]. This work on the male pathway suggests that $K d m 6 b$ can be thought of as a molecular signal that accumulates at cooler temperatures and can initiate testis differentiation by inducing Dmrt 1 expression once a critical threshold is reached. However, this induction of Dmrt1 can be overridden by estrogens as $17 ß$-estradiol inhibits Dmrt1 expression [Murdock and Wibbels, 2006] and results in the development of ovaries (Fig. 4).

\section{Female Pathway}

Although the earliest events in the female pathway are not as well characterized as for the male pathway, there is still a considerable amount known about the genes involved in ovary development. Induction of Forkhead box protein L2 (FoxL2) by warm incubation temperatures $\left(31^{\circ} \mathrm{C}\right)$ is considered a critical regulator of ovary development [Matsumoto and Crews, 2012]; FoxL2 directly interacts with the promoter of aromatase [Govoroun et al., 2004]. Warm incubation temperatures also stimulate demethylation of the aromatase promoter to induce its expression [Matsumoto et al., 2016]. Aromatase is the enzyme responsible for producing estrogens and is necessary for ovary development [Matsumoto and Crews, 


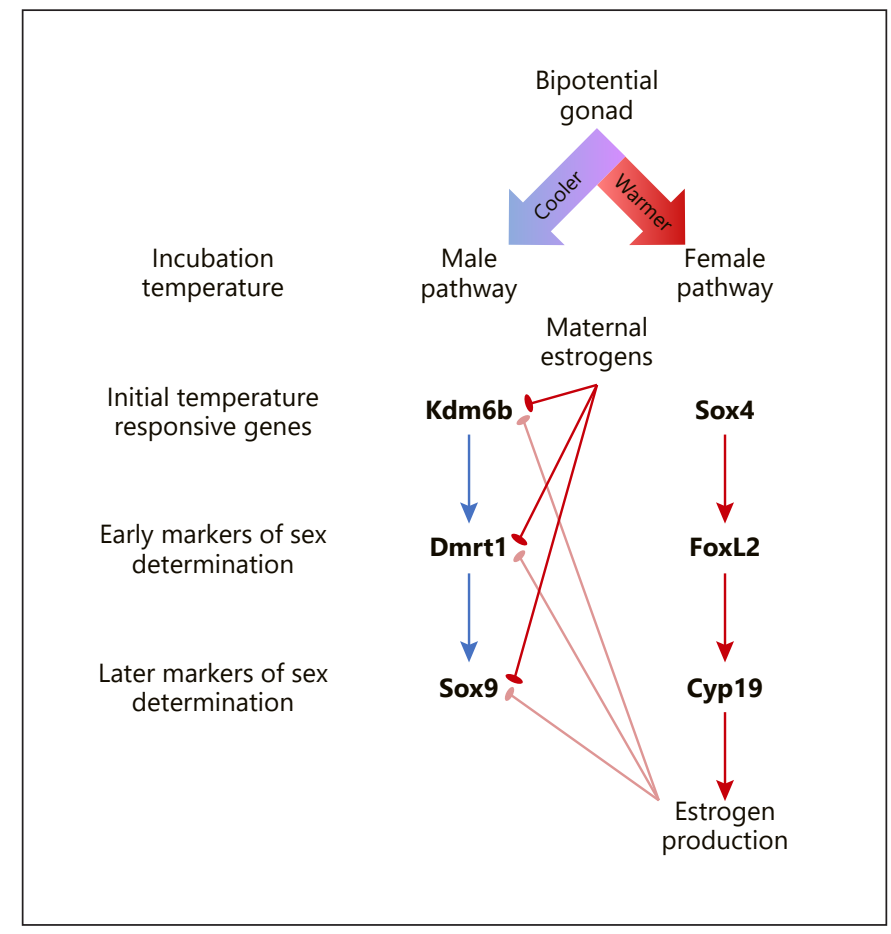

Fig. 4. Proposed model for how estrogens, either maternal or endogenously produced, could affect the responsiveness of the male sex determining pathway.

2012]. Warm temperatures also antagonize the male pathway as there is an influx of calcium at $31^{\circ} \mathrm{C}$ that stimulates the phosphorylation of Stat3, repressing the expression of $K d m 6 b$ and thus reducing the molecular cue for testis differentiation [Weber et al., 2020] (Fig. 4). It remains unknown whether the induction of the female pathway requires a temperature cue independent of the male pathway or if the lack of $K d m 6 b$ and Dmrt 1 at warm temperatures is sufficient for induction of the female pathway. Knockdown of both Kdm6b [Ge et al., 2018] and Dmrt1 [Ge et al., 2017] does result in the development of ovaries.

Collectively, our current understanding of TSD involves temperature affecting processes such as calcium influx, protein phosphorylation, histone methylation/demethylation, DNA methylation/demethylation, and alternative splicing to coordinate the expression of genes necessary for gonadal differentiation. A critical step in this process is whether or not Dmrt1 expression is initiated. But the vast majority of these studies have been conducted using constant thermal conditions, quite unlike what many embryos experience in nature. Little to nothing is known about how these processes operate when

Estrogens, Thermal Responsiveness, and Sex Determination embryos are exposed to the mixed thermal signals present in natural nest conditions or when embryos are exposed to varying endocrine states.

\section{Thermal Responsiveness as a Trait Needing More Attention}

The idea that it can take time for an organism to respond to temperature cues is neither new nor controversial, yet very little work has been done to examine the variation in the exposure necessary to prompt the commitment of gonadal fate. Recent work using the red-eared slider clearly demonstrates that molecular processes [Weber et al., 2020] and sex ratios [Carter et al., 2018; Breitenbach et al., 2020] can respond to very brief exposures to thermal cues, suggesting that there is substantial underlying variation in the exposure duration necessary before gonadal fate is determined. For example, some individuals developed as females after just 5 days of exposure to warm temperatures $\left(29.5 \pm 3^{\circ} \mathrm{C}\right)$ during development, while others, despite 15 days of exposure to the same thermal conditions, developed as males. It is worth noting that $29.5^{\circ} \mathrm{C}$ is by no means an extreme temperature as far as ecological or laboratory conditions go as most studies use continuous exposure to $31^{\circ} \mathrm{C}$ as a female promoting condition. It is conceivable that embryos may commit to the ovarian fate even sooner if exposed to temperatures warmer than $29.5^{\circ} \mathrm{C}$. Regardless, it is clear that some embryos respond to brief thermal cues, while others do not. We have coined this trait "thermal responsiveness" and believe it may be a trait capable of responding to changing climatic conditions.

When considering the brief exposure to thermal cues as occurs in natural nests and attempting to decipher why/how some embryos respond while others do not, there are a number of processes that could be involved. Conceptually, this process often involves a theoretical "threshold" that must be reached for gonadal fate to become committed [Deeming and Ferguson, 1989]. It is possible that embryos that are more thermally responsive simply have a lower threshold than those embryos that do not respond, but it is also possible that responsive embryos accumulate the signal at a faster rate or lose the signal at a slower rate when temperatures change. Defining a single factor that accumulates is also complicated. Despite all we know about how testis differentiation is initiated in T. scripta, it is still not clear if this factor can be defined. Is the signal $K d m 6 b$ transcripts that get translated to a protein that induces Dmrt1 expression [Ge et 
al., 2018]? Is the signal Dmrt1? If we consider $K d m 6 b$ the signal, then responsiveness would be affected by the loss of this signal when embryos experience warm temperatures that drive calcium influx, Stat 3 phosphorylation, possibly intron retention [Deveson et al., 2017], and ultimately inhibition of $K d m 6 b$ expression [Weber et al., 2020]. In reality, the process of triggering gonadal fate likely requires numerous factors to all be present simultaneously, and the removal of any single factor can prohibit the initiation of gonadal differentiation. What we do know about the male pathway in T. scripta highlights how the progression toward a hypothetical threshold to trigger sex can be influenced by the rate of signal accumulation at cool temperatures, as well as the rate of signal loss at warm temperatures [Weber et al., 2020]. This suggests that the progression toward the threshold does not operate as a "ratchet" mechanism; signals can be lost as well. Additional evidence suggesting that signal loss occurs comes from our work on heatwave continuity, where small breaks in heat exposure decrease the number of females produced despite all embryos receiving identical amounts of heat exposure over a relatively short period during the TSP [Breitenbach et al., 2020]. With regard to thermal responsiveness, variation in any of these processes related to signal gain and loss could underlie variation in the responsiveness of the embryo to thermal cues.

\section{Are Maternal Estrogens Really Important?}

While it is clear that when eggs experience consistently warm or cool incubation conditions throughout the TSP in either the field or the laboratory, those thermal conditions exert a strong effect on sex determination that overrides the effects of maternal steroids (Fig. 3). So, does this mean that maternal estrogens only play a minor role in sex determination? No. Natural conditions are almost certain to provide intermediate thermal cues for sex determination as a result of transient fluctuations of temperature above and below $\mathrm{T}_{\text {piv. }}$. It is increasingly clear that under conditions with transient thermal exposures, small differences in the timing or duration of exposure can have strong effects on sex ratios. Whether it is a shift of only a few days in the timing of heat exposure [Breitenbach et al., 2020], a short extension of a heatwave [Carter et al., 2018], or a short break in heat exposure [Breitenbach et al., 2020], these small changes dramatically shift sex ratios. From the perspective of the underlying mechanism, this suggests that gonads are frequently on the cusp of committing to the gonadal fate, and small shifts in signal accumulation or loss (i.e., a few days more or less) can be the difference between developing testes or ovaries.

Many of the genes involved in the male pathway are subject to inhibition by estradiol (Fig. 4), while others in the female pathway are subject to induction by estradiol. If maternal estrogens, which are present at oviposition and are elevated in late season eggs, have similar effects on the expression of these genes, it suggests that eggs with elevated estrogens should require less exposure to warm temperatures to induce ovarian differentiation, as we have already demonstrated in T. scripta [Carter et al., 2018]. At this point, it is not clear how direct the effects of estrogens are on the expression of different genes in the sex determining cascade. It is plausible that the expression of upstream genes such as $K d m 6 b$ are directly responding to activated estrogen receptors and subsequently regulating genes downstream. Transcriptomic analyses suggest that hormonal regulation of epigenetic processes is possible in species with TSD [Radhakrishnan et al., 2018]. Alternatively, downstream genes such as Dmrt1 may directly respond to activated estrogen receptors and allow estrogens to override the effects $K d m 6 b$ has on Dmrt1 expression [Ge et al., 2018]. It is also likely that the effects of estradiol do not operate via identical mechanisms as estrone sulfate resulting in more complexity in terms of how maternal estrogens might regulate genes involved in gonadal differentiation. Now, we must close the circle by examining gene expression patterns in eggs with elevated estrogens to determine if maternal estrogens can hinder the cascade that would result in testis formation (Fig. 4).

\section{Evolutionary Implications}

Conceptually, thermal responsiveness is a relatively straightforward trait where there is variation in the amount of time it takes for an organism to respond to a thermal cue. However, the mechanisms underlying this variation are likely quite intricate and involve both genetic and plastic components. Adding to the complexity and variation related to natural incubation temperatures [Valenzuela et al., 2019; Bock et al., 2020a], it becomes clear that there is much to learn about thermal responsiveness. As a trait, thermal responsiveness may be relevant to any physiological process that responds to environmental temperatures, with TSD being a process deserving urgent attention given the obvious implications of altered sex ratios in response to changing climatic conditions. Recent advances in our understanding of some of 
the initial responses of the gonad to incubation temperatures in species with TSD now allow us to investigate variation in the timing of this response [Czerwinski et al., 2016; Yatsu et al., 2016; Deveson et al., 2017; Radhakrishnan et al., 2017; Ge et al., 2018]. Even when focusing on reptile species with TSD, the complexities of thermal responsiveness quickly emerge. For example, both American alligators (Alligator mississippiensis) [Yatsu et al., 2016] and red-eared sliders [Czerwinski et al., 2016] exhibit alternative splicing of $K d m 6 b$ at cooler incubation temperatures [Deveson et al., 2017], but in alligators the temperature at which this intron retention occurs is female producing [Bock et al., 2020b] whereas in turtles this temperature is male producing [Deveson et al., 2017]. This suggests that the response of splicing to temperature may be similar in the 2 species, but the resulting histone demethylation by $K d m 6 b$ could be different. Genetic variation that affects processes like RNA splicing and histone demethylation could drive variation in thermal responsiveness. Given the multifaceted nature of how incubation temperature is integrated into a cue for sex determination, there is likely ample genetic variation underlying variation in thermal responsiveness that could evolve in response to selective pressures as a result of changing climatic conditions. In a scenario where incubation temperatures become warmer over time (i.e., higher max temperatures, longer heatwaves, higher nighttime temperatures), we hypothesize that becoming less responsive to warm conditions or more responsive to cooler conditions would allow organisms to adapt. By requiring either more time at warm conditions or less time at cool conditions before committing to the gonadal fate, species may be able to maintain more stable sex ratios in response to changing climatic conditions. Empirical tests of this hypothesis are now necessary and should include a range of species that possess different patterns of TSD and inhabit different climates. Understanding how species have adapted to their current climates will substantially improve our ability to predict how they may respond to future climates.

Many of the genes affected by incubation temperatures in species with TSD are also regulated by steroids [Ramsey and Crews, 2009; Matsumoto et al., 2013; Kohno et al., 2020], creating the potential for steroid-mediated maternal effects to influence thermal responsiveness. And while thermal responsiveness may be a trait that ranges across many taxa, the number of species where maternal steroids may affect thermal responsiveness is less clear. It is also unclear how estrogenic endocrine disrupting compounds (EDCs) may affect thermal responsiveness, but it is pos-

Estrogens, Thermal Responsiveness, and Sex Determination sible that estrogenic EDCs could modify the response to natural conditions that might otherwise be attributed to maternal steroids [Clairardin et al., 2013; Paitz and Bowden, 2015; Mizoguchi and Valenzuela, 2016]. Not all thermally responsive processes are likely sensitive to steroids, but in the particular case of TSD, it is clear that sex determination is sensitive to steroids [Wibbels et al., 1991b; Kohno et al., 2015]. Surprisingly, relatively little work has been done on how maternal steroids influence sex determination [Radder, 2007; Bowden and Paitz, 2018]. While there is some evidence that yolk steroids such as corticosterone [Warner et al., 2009] and dihydrotestosterone [Radder et al., 2009] may influence sex ratios in species with TSD, the vast majority of data in this area focuses on estrogens [Bowden et al., 2000; Carter et al., 2017]. These studies demonstrate repeatable seasonal shifts in yolk estrogens across several species of turtles, with the elevated estrogen levels present in late season eggs being associated with the increased production of female hatchlings at intermediate temperatures [Bowden et al., 2000, 2011, 2014; Paitz and Bowden, 2009, 2013; Carter et al., 2018]. Whether there is an adaptive significance to this seasonal shift remains to be studied, but it is worth noting that most of the empirical support that exists for the adaptive significance of TSD in reptiles does involve a seasonal shift in sex ratios [Warner and Shine, 2008]. It is also worth noting that these studies focus on populations of North American turtles from the northern end of the distribution range [Ernst and Lovich, 2009]. Given that females are produced at warmer temperatures in these species, it is plausible that cooler northern climates select for processes that are likely to increase the production of female hatchlings. It remains unknown whether turtles from southern populations or species that have differing patterns of TSD exhibit a seasonal increase in maternal estrogens. Understanding whether maternal effects currently influence how populations in different climates persist will improve our ability to predict the role of maternal effects in future climates.

\section{Conclusions}

TSD has been an area of fascination for researchers for well over 50 years, yet it is clear that we are just beginning to understand the complex network of factors that transduce the thermal cue into a molecular signal that results in the formation of the gonad. More work using ecologically relevant thermal cues (i.e., brief exposures to heat and cold) is needed to decipher how maternal estrogens 
affect sex determination under these intermediate thermal cues. Such studies will help to clarify the key factors that are driving sex determination in the field and may prove critical for conservation efforts as many species with TSD are likely to suffer sex ratio biases as climatic conditions change.

\section{Acknowledgement}

We would like to thank the many students that contributed to the research program over the years.

\section{Conflict of Interest Statement}

The authors have no conflicts of interest to declare.

\section{Funding Sources}

The authors have no funding sources to declare.

\section{Author Contributions}

R.M.B. and R.T.P. contributed equally to the development and writing of this manuscript.

\section{References}

Abe Y, Fujiwara Y, Takahashi H, Matsumura Y, Sawada T, Jiang S, et al. Histone demethylase JMJD1A coordinates acute and chronic adaptation to cold stress via thermogenic phospho-switch. Nat Commun. 2018;9(1):1566.

Amano T, Smithers RJ, Sparks TH, Sutherland WJ, A. A 250-year index of first flowering dates and its response to temperature changes. Proc Biol Sci. 2010;277(1693):2451-7.

Anand A, Patel M, Lalremruata A, Singh AP, Agrawal R, Singh L, et al. Multiple alternative splicing of Dmrtl during gonadogenesis in Indian mugger, a species exhibiting temperature-dependent sex determination. Gene. 2008;425(1-2):56-63.

Bachtrog D, Mank JE, Peichel CL, Kirkpatrick M, Otto SP, Ashman TL, et al. Sex determination: Why so many ways of doing it? PLoS Biol. 2014;12(7):e1001899-13.

Bock SL, Lowers RH, Rainwater TR, Stolen E, Drake JM, Wilkinson PM, et al. Spatial and temporal variation in nest temperatures forecasts sex ratio skews in a crocodilian with environmental sex determination. Proc Biol Sci. 2020a;287(1926):20200210.

Bock SL, Hale MD, Leri FM, Wilkinson PM, Rainwater TR, Parrott BB. Post-transcriptional mechanisms respond rapidly to ecologically relevant thermal fluctuations during temperature-dependent sex determination. Integr Org Biol. 2020b;2(1):obaa033.

Bowden RM, Paitz RT. Temperature fluctuations and maternal estrogens as critical factors for understanding temperature-dependent sex determination in nature. J Exp Zool. 2018; 329(4-5):177-84.

Bowden RM, Ewert MA, Nelson CE. Environmental sex determination in a reptile varies seasonally and with yolk hormones. Proc Biol Sci. 2000;267(1454):1745-9.

Bowden RM, Ewert MA, Lipar JL, Nelson CE. Concentrations of steroid hormones in layers and biopsies of chelonian egg yolks. Gen Comp Endocrinol. 2001;121(1):95-103.
Bowden RM, Paitz RT, Janzen FJ. The ontogeny of postmaturation resource allocation in turtles. Physiol Biochem Zool. 2011;84(2):20411.

Bowden RM, Carter AW, Paitz RT. Constancy in an inconstant world: moving beyond constant temperatures in the study of reptilian incubation. Integr Comp Biol. 2014;54(5):83040.

Breitenbach AT, Carter AW, Paitz RT, Bowden RM. Using naturalistic incubation temperatures to demonstrate how variation in the timing and continuity of heat wave exposure influences phenotype: Heat waves affect phenotype. Proc R Soc B Biol Sci. 2020;287(1932): 20200992.

Bull JJ. Sex determination in reptiles. Q Rev Biol. 1980;55(1):3-21.

Bull JJ, Vogt RC, McCoy CJ. Sex determining temperatures in turtles: A geographic comparison. Evolution. 1982;36(2):326-32.

Capel B. Vertebrate sex determination: Evolutionary plasticity of a fundamental switch. Nat Rev Genet. 2017;18(11):675-89.

Capovilla G, Symeonidi E, Wu R, Schmid M. Contribution of major FLM isoforms to temperature-dependent flowering in Arabidopsis thaliana. J Exp Bot. 2017;68(18):5117-27.

Carter AL, Bodensteiner BL, Iverson JB, MilneZelman CL, Mitchell TS, Refsnider JM, et al. Breadth of the thermal response captures individual and geographic variation in temperature-dependent sex determination. Funct Ecol. 2019;33(10):1928-39.

Carter AW, Bowden RM, Paitz RT. Seasonal shifts in sex ratios are mediated by maternal effects and fluctuating incubation temperatures. Funct Ecol. 2017;31(4):876-84.

Carter AW, Sadd BM, Tuberville TD, Paitz RT, Bowden RM. Short heatwaves during fluctuating incubation regimes produce females under temperature-dependent sex determination with implications for sex ratios in nature. Sci Rep. 2018;8(1):3-13.
Carter AW, Paitz RT, Bowden RM. The devil is in the details: Identifying aspects of temperature variation that underlie sex determination in species with TSD. Integr Comp Biol. 2019; 59(4):1081-8.

Clairardin SG, Paitz RT, Bowden RM. In ovo inhibition of steroid metabolism by bisphenol-A as a potential mechanism of endocrine disruption. Proc Biol Sci. 2013;280(1769):20131773.

Czerwinski M, Natarajan A, Barske L, Looger LL, Capel B. A timecourse analysis of systemic and gonadal effects of temperature on sexual development of the red-eared slider turtle Trachemys scripta elegans. Dev Biol. 2016; 420(1):166-77.

Deeming DC, Ferguson MWJ. The mechanism of temperature dependent sex determination in crocodilians: A hypothesis. Am Zool. 1989; 29(3):973-85.

Deveson IW, Holleley CE, Blackburn J, Marshall Graves JA, Mattick JS, Waters PD, et al. Differential intron retention in Jumonji chromatin modifier genes is implicated in reptile temperature-dependent sex determination. Sci Adv. 2017;3(6):e1700731.

Ernst C, Lovich J. Turtles of the United States and Canada. Baltimore, MD: Johns Hopkins University Press; 2009.

Fleming KA, Perrault JR, Stacy NI, Coppenrath CM, Gainsbury AM. Heat, health and hatchlings: associations of in situ nest temperatures with morphological and physiological characteristics of loggerhead sea turtle hatchlings from Florida. Conserv Physiol. 2020;8(1):coaa046-17.

Ge C, Ye J, Zhang H, Zhang Y, Sun W, Sang Y, et al. induces the male pathway in a turtle species with temperature-dependent sex determination. Development. 2017;144(12):2222-33.

Ge C, Ye J, Weber C, Sun W, Zhang H, Zhou Y, et al. The histone demethylase KDM6B regulates temperature-dependent sex determination in a turtle species. Science. 2018;360(6389):645-8.

Georges A. Female turtles from hot nests: is it duration of incubation or proportion of development at high temperatures that matters? Oecologia. 1989;81(3):323. 
Georges A, Limpus C, Stoutjesdijk R. Hatchling sex in the marine turtle Caretta caretta is determined by proportion of development at a temperature, not daily duration of exposure. J Exp Zool. 1994;270(5):432-44.

Gómez-Saldarriaga C, Valenzuela N, Ceballos CP. Effects of incubation temperature on sex determination in the endangered Magdalena river turtle, Podocnemis lewyana. Chelonian Conserv Biol. 2016;15:43-53.

Govoroun MS, Pannetier M, Pailhoux E, Cocquet J, Brillard JP, Couty I, et al. Isolation of chicken homolog of the FOXL2 gene and comparison of its expression patterns with those of aromatase during ovarian development. Dev Dyn. 2004;231(4):859-70.

Hall JM, Warner DA. Ecologically relevant thermal fluctuations enhance offspring fitness: biological and methodological implications for studies of thermal developmental plasticity. J Exp Biol. 2020;223(Pt 19):231902.

Kinmonth-Schultz HA, MacEwen MJS, Seaton DD, Millar AJ, Imaizumi T, Kim S-H. An explanatory model of temperature influence on flowering through whole-plant accumulation of flowering locus $\mathrm{T}$ in Arabidopsis thaliana. Silico Plants. 2019;1(1):1-22.

Kohno S, Bernhard MC, Katsu Y, Zhu J, Bryan TA, Doheny BM, et al. Estrogen receptor 1 (ESR1; ER $\alpha$ ), not ESR2 (ER $\beta)$, modulates estrogen-induced sex reversal in the American alligator, a species with temperature-dependent sex determination. Endocrinology. 2015; 156(5):1887-99.

Kohno S, Vang D, Ang E, Brunell AM, Lowers RH, Schoenfuss HL. Estrogen-induced ovarian development is time-limited during the temperature-dependent sex determination of the American alligator. Gen Comp Endocrinol. 2020;291:113397.

Lambeth LS, Raymond CS, Roeszler KN, Kuroiwa A, Nakata T, Zarkower D, et al. Over-expression of DMRT1 induces the male pathway in embryonic chicken gonads. Dev Biol. 2014; 389(2):160-72.

Les HL, Paitz RT, Bowden RM. Experimental test of the effects of fluctuating incubation temperatures on hatchling phenotype. J Exp Zool A Ecol Genet Physiol. 2007;307(5):274-80.

Les HL, Paitz RT, Bowden RM. Living at extremes: Development at the edges of viable temperature under constant and fluctuating conditions. Physiol Biochem Zool. 2009; 82(2):105-12.

Matsumoto Y, Crews D. Molecular mechanisms of temperature-dependent sex determination in the context of ecological developmental biology. Mol Cell Endocrinol. 2012;354(1-2): 103-10.

Matsumoto Y, Hannigan B, Crews D. Temperature shift alters DNA methylation and histone modification patterns in gonadal aromatase (cyp19a1) gene in species with temperaturedependent sex determination. PLoS One. 2016;11(11):e0167362.
Matsumoto Y, Yatsu R, Taylor C, Crews D. Changes in gonadal gene network by exogenous ligands in temperature-dependent sex determination. J Mol Endocrinol. 2013;50(3): 389-400.

Mizoguchi BA, Valenzuela N. Ecotoxicological perspectives of sex determination. Sex Dev. 2016;10(1):45-57.

Mork L, Czerwinski M, Capel B. Predetermination of sexual fate in a turtle with temperature-dependent sex determination. Dev Biol. 2014;386(1):264-71.

Mrosovsky N, Pieau C. Transitional range of temperature, pivotal temperatures and thermosensitive stages for sex determination in reptiles. Amphib Reptil. 1991;12:169-79.

Murdock C, Wibbels T. Dmrt1 expression in response to estrogen treatment in a reptile with temperature-dependent sex determination. J Exp Zool B Mol Dev Evol. 2006;306(2):134-9.

Paitz RT, Bowden RM. Rapid decline in the concentrations of three yolk steroids during development: Is it embryonic regulation? Gen Comp Endocrinol. 2009;161(2):246-51.

Paitz RT, Bowden RM. Biological activity of oestradiol sulphate in an oviparous amniote: Implications for maternal steroid effects. Proc Biol Sci. 2011;278(1714):2005-10.

Paitz RT, Bowden RM. Sulfonation of maternal steroids is a conserved metabolic pathway in vertebrates. Integr Comp Biol. 2013;53(6): 895-901.

Paitz RT, Bowden RM. The in ovo conversion of oestrone to oestrone sulfate is rapid and subject to inhibition by bisphenol a. Biol Lett. 2015;11(4):20140946.

Paitz RT, Clairardin SG, Griffin AM, Holgersson MCN, Bowden RM. Temperature fluctuations affect offspring sex but not morphological, behavioral, or immunological traits in the Northern Painted Turtle (Chrysemys picta). Can J Zool. 2010;88(5):479-86.

Paitz RT, Sawa AR, Bowden RM. Characterizing the metabolism and movement of yolk estradiol during embryonic development in the red-eared slider (Trachemys scripta). Gen Comp Endocrinol. 2012;176(3):507-12.

Politis SN, Mazurais D, Servili A, Zambonino-Infante JL, Miest JJ, Sørensen SR, et al. Temperature effects on gene expression and morphological development of European eel, Anguilla anguilla larvae. PLoS One. 2017;12(8):1-23.

Preußner M, Goldammer G, Neumann A, Haltenhof T, Rautenstrauch P, Müller-McNicoll M, et al. Body temperature cycles control rhythmic alternative splicing in mammals. Mol Cell. 2017;67(3):433-46

Radder RS. Maternally derived egg yolk steroid hormones and sex determination: review of a paradox in reptiles. J Biosci. 2007;32(6):121320.

Radder RS, Pike DA, Quinn AE, Shine R. Offspring sex in a lizard depends on egg size. Curr Biol. 2009;19(13):1102-5.
Radhakrishnan S, Literman R, Neuwald J, Severin A, Valenzuela N. Transcriptomic responses to environmental temperature by turtles with temperature-dependent and genotypic sex determination assessed by RNAseq inform the genetic architecture of embryonic gonadal development. PLoS One. 2017;12(3): e0172044.

Radhakrishnan S, Literman R, Neuwald JL, Valenzuela N. Thermal response of epigenetic genes informs turtle sex determination with and without sex chromosomes. Sex Dev. 2018.doi: $10.1159 / 000492188$

Ramsey M, Crews D. Steroid signaling and temperature-dependent sex determination-Reviewing the evidence for early action of estrogen during ovarian determination in turtles. Semin Cell Dev Biol. 2009;20(3):283-92.

Shipley JR, Twining CW, Taff CC, Vitousek MN, Flack A, Winkler DW. Birds advancing lay dates with warming springs face greater risk of chick mortality. Proc Natl Acad Sci USA. 2020;117(41):25590-4.

Valenzuela N. Constant, shift, and natural temperature effects on sex determination in Podocnemis expansa turtles. Ecology. 2001; 82(11):3010-24.

Valenzuela N, Literman R, Neuwald JL, Mizoguchi B, Iverson JB, Riley JL, et al. Extreme thermal fluctuations from climate change unexpectedly accelerate demographic collapse of vertebrates with temperature-dependent sex determination. Sci Rep. 2019;9(1):4254-11.

Visser ME, Both C. Shifts in phenology due to global climate change: The need for a yardstick. Proc Biol Sci. 2005;272(1581):2561-9.

Warner DA, Shine R. The adaptive significance of temperature-dependent sex determination in a reptile. Nature. 2008;451(7178):566-8.

Warner DA, Radder RS, Shine R. Corticosterone exposure during embryonic development affects offspring growth and sex ratios in opposing directions in two lizard species with environmental sex determination. Physiol Biochem Zool. 2009;82(4):363-71.

Weber C, Zhou Y, Lee JG, Looger LL, Qian G, Ge $\mathrm{C}$, et al. Temperature-dependent sex determination is mediated by pSTAT3 repression of Kdm6b. Science. 2020;368(6488):303-6.

Wibbels T, Bull JJ, Crews D. Chronology and morphology of temperature-dependent sex determination. J Exp Zool. 1991a;260(3):37181.

Wibbels T, Bull JJ, Crews D. Synergism between temperature and estradiol: A common pathway in turtle sex determination? J Exp Zool. 1991b;260(1):130-4.

Yatsu R, Miyagawa S, Kohno S, Parrott BB, Yamaguchi K, Ogino Y, et al. RNA-seq analysis of the gonadal transcriptome during Alligator mississippiensis temperature-dependent sex determination and differentiation. BMC Genomics. 2016;17:77-13.

Zhao L, Svingen T, Ng ET, Koopman P. Femaleto-male sex reversal in mice caused by transgenic overexpression of Dmrt1. Development. 2015;142(6):1083-8. 